\title{
Multiperiodic RR Lyrae stars in the field of NGC 6362
}

\author{
Paweł Moskalik ${ }^{1, \star}$, Radosław Smolec ${ }^{1}$, Janusz Kałużny ${ }^{1 \star \star}$, Wojciech Pych ${ }^{1}$, Michał Różyczka ${ }^{1}$, \\ and Ian B. Thompson ${ }^{2}$ \\ ${ }^{1}$ Nicolaus Copernicus Astronomical Center, Warsaw, Poland \\ ${ }^{2}$ The Observatories of the Carnegie Institution for Science, Pasadena, USA
}

Abstract. We present frequency analysis of RR Lyrae stars of globular cluster NGC 6362.

\section{Observations and data analysis}

NGC 6362 is a nearby globular cluster of Oosterhoff I type. It has a mean metallicity of $[\mathrm{Fe} / \mathrm{H}]=-0.95$ and belongs to the old halo population ([1]). 35 RR Lyrae-type stars have been discovered in the cluster ([5]). We revisit these variables, taking advantage of the new CCD photometry accumulated by the Cluster AgeS Experiment (CASE). In our analysis we used $3200 \mathrm{~V}$-band images collected with the 1-m Swope telescope of the Las Campanas Observatory between July 8th, 1999 and September 9th, 2009. The frequency analysis of the data was performed with the standard consecutive prewhitening technique. For full discussion of our results we refer the reader to [6].

\section{RR Lyrae variables of NGC 6362}

16 RR Lyrae stars of NGC 6362 are fundamental mode pulsators (RRab variables) and 16 are first overtone pulsators (RRc variables). Two objects, previously identified as RRab stars, turned out to be double mode pulsators (RRd stars), with two lowest radial modes simultaneously excited. Finally, in V37 we detect two high-amplitude modes of close frequencies and with lightcurves significantly differing from those of RR Lyrae stars. The nature of V37 is a puzzle. It will not be discussed here.

\subsection{Non-radial modes}

In 10 out of 16 RRc stars of NGC 6362 (63\% of the sample) we detect a secondary short period variability, with the period ratio falling in the range of $P_{\mathrm{x}} / P_{1}=0.60-0.65$. The amplitudes of the secondary signals never exceed 6 mmag. Similar pulsators have recently been discovered in many stellar systems ([3], and references therein). Space observations indicate that this form of variability most likely occurs in almost all RRc and RRd stars ([4]). Detecting it in the ground-based data is difficult, however, because of the extremely low amplitude of the secondary periodicities. The incidence rate of $63 \%$, that we report for NGC 6362, is the highest among all stellar systems observed

\footnotetext{
$\star_{\text {mailto:pam@camk.edu.pl }}$

$\star \star$ deceased
} 
from the ground. The properties of the stars identified in NGC 6362 are qualitatively similar to those discovered in the Galactic bulge. In particular, in the period ratio vs. period diagram (so called Petersen diagram) they fall within the three sequences defined by the Galactic bulge stars.

The period ratios of $0.60-0.65$ cannot be explained by two radial modes. This implies that the secondary periodicities observed in the RRc stars must be due to non-radial modes of oscillation. In the model recently proposed by Dziembowski ([2]), these periodicities are identified with the harmonics of modes of angular degrees of $\ell=8$ and $\ell=9$. In this picture, the non-radial modes themselves have amplitudes lower than their harmonics, because of geometric cancelation effects.

\subsection{The Blazhko effect}

The Blazhko effect is a periodic (or almost periodic) modulation of pulsation amplitudes and phases. In the frequency domain it manifests itself as a presence of equally spaced triplets (or higher multiplets) centered on the pulsation frequency and its harmonics. Sometimes only a doublet is detected, with the other component of the triplet hidden in the noise. The separation of components within the multiplet corresponds to the modulation frequency. We have found the Blazhko effect in 11 RRab and in 3 RRc stars of NGC 6362. This corresponds to incidence rates of $69 \%$ and 19\%, respectively, which are among the highest reported in ground-based observations. The observed modulation periods range from 17.3 day to 216.4 day for RRab stars and from 8.5 day to 15.5 day for RRc stars. In one RRc and in one RRab star we find a rare double-periodic Blazhko effect. The weakest modulation detected in NGC 6362 has a peak-to-peak amplitude of only 7 mmag (in the RRc star V36).

\subsection{Anomalous RRd variables}

Two stars previously classified as RRab variables, V3 and V34, turned out to be double mode pulsators. In addition to the dominant radial fundamental mode, we also detect the radial first overtone of very low amplitude. The period ratios for the two modes in V3 and V34 are 0.730 and 0.728 , respectively. These values are significantly lower than for the majority of the "classical" RRd variables of the same fundamental mode period. In both stars we detect the Blazhko effect, with either the fundamental mode only (V34) or both radial modes (V3) being modulated. Lower than usual period ratios, strong domination of the radial fundamental mode and presence of a long-period Blazhko modulation all indicate that V3 and V34 are not typical RRd stars. They must be classified as new members of the recently identified group of anomalous RRd variables ([7]).

Acknowledgments: This research is supported by the Polish National Science Center, through grant DEC2015/17/B/ST9/03421

\section{References}

[1] Catelan, M., Ap\&SS, 320, 261 (2009)

[2] Dziembowski, W., Comm. Konkoly Observatory, 105, 23 (2016)

[3] Netzel, H., Smolec, R., \& Moskalik, P., MNRAS, 453, 2022 (2015)

[4] Moskalik, P., Smolec, R., Kolenberg, K. et al., MNRAS, 447, 2348 (2015)

[5] Olech, A., Kałużny, J., Thompson, I., Pych, W., Krzemiński, W., \& Schwarzenberg-Czerny, A., MNRAS, 321, 421 (2001)

[6] Smolec, R., Moskalik, P., Kałużny, J., Pych, W., Różyczka, M., \& Thompson, I. B., MNRAS, 467, 2349 (2017)

[7] Soszyński, I., Smolec, R., Dziembowski, W., et al., MNRAS, 463, 1332 (2016) 